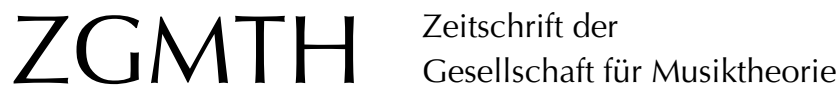

Krämer, Laura (2007): Fauxbourdon bei Bartók. ZGMTH 4/1-2, 123-134.

https://doi.org/10.31751/248

() 2007 Laura Krämer

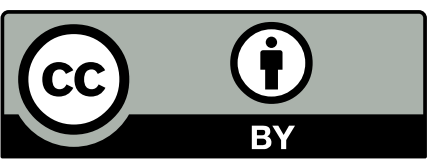

Dieser Text erscheint im Open Access und ist lizenziert unter einer Creative Commons Namensnennung 4.0 International Lizenz.

This is an open access article licensed under a

Creative Commons Attribution 4.0 International License.

veröffentlicht / first published: 01/01/2007

zuletzt geändert / last updated: 08/04/2009 


\title{
Fauxbourdon bei Bartók
}

\author{
Laura Krämer
}

Das Mixturprinzip ist in Bartóks Werk eine bedeutende Klangtechnik. Unter allen möglichen Mixturen hat die Sextakkordmixtur eine Sonderstellung inne, da sie als `Fauxbourdon geschichtlich besetzt ist. Anhand der Sonate für zwei Klaviere und Schlagzeug und dem Konzert für Orchester werden zwei verschiedene Möglichkeiten gezeigt, mit Sextakkordmixturen umzugehen: In der Sonate für zwei Klaviere inszeniert Bartók den Fauxbourdon als dolceCharakter, bezugnehmend auf die semantische Konnotation der Fauxbourdon-Figur. Typische Verfremdungsstrategien (reale Mixtur, dissonierende Haltetöne etc.) sind zu beobachten. Im letzten Satz des Konzerts für Orchester ergibt sich die Sextakkordmixtur wie neu erfunden aus dem Imitationsschema der Violinen; ihr Gestus unterscheidet sich deutlich vom traditionellen Fauxbourdon-Charakter. An demselben Werk lässt sich belegen, dass im 20. Jahrhundert die Quartsextakkord-Mixtur als dolce-Charakter die verbrauchte Sextakkordmixtur ablöst.

Bartóks Werk steht an der Nahtstelle zwischen der traditionellen europäischen Kompositionsgeschichte und der Neuen Musik. In vielerlei Hinsicht fungierte Bartók als Mittler, durch den hergebrachte musikalische Materialien und Verarbeitungstechniken in die Zukunft transportiert wurden. Der Vorgang ist nicht selbstverständlich, denn alte Kompositionsweisen hatten in der Musik nach 1945, die den radikalen Neuanfang suchte, einen schweren Stand. Dass Bartóks Traditionsverbundenheit dennoch akzeptiert wurde, lag an dem radikalen Eklektizismus, mit dem er alte Verfahrensweisen aufgriff und mit neuen kombinierte: So bewahrte er sich ein hohes Maß künstlerischer Ungebundenheit, ja strenggenommen war Bartóks kreative Freiheit sogar größer als jene von Komponisten, die den Bezug auf schon Dagewesenes für ihr Werk ausschlossen. 1931 äußerte er: »Auch in der Musik, wie in der Literatur oder den bildenden Künsten ist es ganz bedeutungslos, welchen Ursprungs das verarbeitete Thema ist, wichtig ist aber die Art, wie wir es verarbeiten. In diesem `Wie ‘ offenbart sich das Können, die Gestaltungs- und Ausdruckskraft, die Persönlichkeit des Künstlers. « ${ }^{1}$

Zur horizontalen und vertikalen Tonhöhenorganisation benutzte Bartók verschiedenste traditionelle, volkstümliche oder symmetrisch konstruierte Skalen, Rhythmen, Mixtur- und Kanonprinzipien, aber auch experimentelle Techniken wie Cluster, Klangflecken, Mikrotonalität.

Unter Bartóks Mixturen finden sich neben Quartenakkorden und stark dissonanten Gebilden auch alle Umkehrungen des Dreiklangs. Der Sextakkord nimmt hier zwangs-

1 Bartók 1931, Szabolcsi 1972, 175, zit. nach Fladt 1996, 6. 
läufig eine Sonderstellung ein: Er ist die einzige Kopplung dreier Stimmen, deren Parallelführung auch unter Einhaltung der alten Kontrapunktregeln möglich war. ${ }^{2}$ Zu Bartóks Zeit blickte die Sextakkordmixtur unter dem Namen `Fauxbourdon` bereits auf eine fünfhundertjährige Geschichte, erst als Kontrapunkt-Technik, dann als semantisch konnotierte Figur, zurück. Aufgrund ihrer Tradition und ihres charakteristischen Klanges kann sie bei Bartók niemals ohne Verfremdung eingesetzt werden. So bevorzugt Bartók reale anstelle von tonalen bzw. modalen Mixturen. Auf diese Weise fügen sich die konsonanten Klangerscheinungen dennoch nicht zu einem Dur-Moll-tonalen Gesamtraum zusammen.

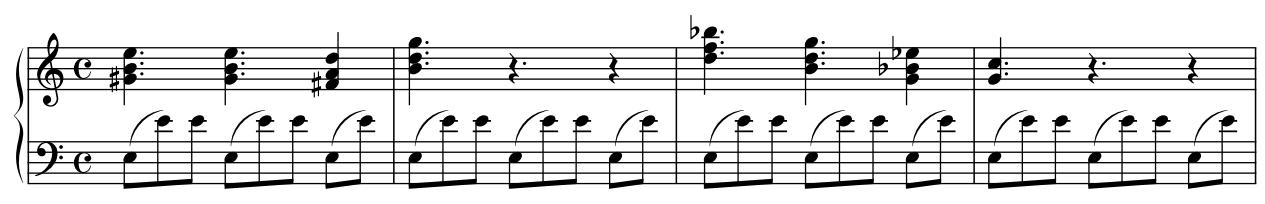

Beispiel 1: Béla Bartók, Tanz im bulgarischen Rhythmus (Mikrokosmos Bd. 6, Nr. 153). Reale Mixtur von Dur-Sextakkorden.

Zwei Werke sollen hier Gegenstand genauerer Betrachtungen sein, und zwar der erste Satz der Sonate für zwei Klaviere und Schlagzeug (1937) und zwei Sätze aus dem Konzert für Orchester (1945). Der Umgang mit dem Fauxbourdon ist in diesen beiden Werken recht gegensätzlich, so dass, auch wenn hier viele andere Belegstücke außen vor bleiben müssen, am Ende ein Eindruck davon entstehen sollte, welche Bandbreite ein einziger musikalischer Topos ${ }^{3}$ auf seinem Weg ins 20. Jahrhundert gewinnen kann.

\section{Sonate für zwei Klaviere und Schlagzeug}

Musikalische Elemente des Fauxbourdon (die Intervalle Quarte und Sexte sowie das Mixtur- und das Kanonprinzip) spielen in diesem formal an die Sonatenform Beethovens angelehnten Satz eine so elementare Rolle, dass er sich zeitweilig als Komposition über die musikalische Idee des `Fauxbourdon` lesen lässt.

Reale Dur-Sextakkordmixturen charakterisieren das Thema des Seitensatzes. Im Verlauf des Satzes taucht es dreimal auf, zweimal in der Exposition (T. $84 \mathrm{ff}$. und T. 161) und einmal in der Reprise (T. 292). Das Thema verläuft im Tempo stranquillo in (teils punktierten) Halben und Vierteln und trägt die Vorschrift piano, dolce. So hebt es sich deutlich vom schnellen, motorischen und lauten Rest des Satzes ab. Mit dieser Charakterisierung stellt sich Bartók in die Tradition der musikalisch-rhetorischen Figur des

2 Parallelen von perfekten Konsonanzen sind unerwünscht, und Dissonanzen können in Parallelführung nicht korrekt behandelt werden. Im `Fauxbourdon` ergeben sich zur untersten Stimme die imperfekten Konsonanzen Terz und Sext. Die Quarten zwischen Ober- und Mittelstimme erhalten durch die darunter liegende dritte Stimme ebenfalls den Status von imperfekten Konsonanzen und können so parallelgeführt werden.

3 Der Toposbegriff wurde von Ernst Robert Curtius literaturgeschichtlich eingeführt. Zum musikalischen Toposbegriff siehe Jeßulat 2000. 
'Fauxbourdon`, die unter anderem mit Weichheit assoziiert war. ${ }^{4}$ Drei Aspekte sind in der Analyse der Fauxbourdonpartien wesentlich: Erstens die traditionellen Anteile, zweitens die Verfremdungsstrategien und drittens die Integration in den musikalischen Gesamtzusammenhang. Die historischen Anteile liegen in allen drei Stellen im parallelen Verschieben von Sextakkorden und in der sweichen Inszenierung des Fauxbourdon. Eine Verfremdungsstrategie besteht an allen drei Stellen in der Verwendung der realen Mixtur von Durakkorden, so dass die tonalen Assoziationen, die durch die konsonanten Akkorde hervorgerufen werden, ohne Entsprechung im sich ergebenden Tonvorrat bleiben. Außerdem werden die Fauxbourdonpartien von Tönen begleitet, die als Tonrepetition (1. Stelle) oder als Umspielung einer immer gleichen Tonhöhe (2. und 3. Stelle) eine starre dissonierende Achse bilden.

Beim ersten Auftreten T. 84 ff. bilden die Melodietöne jeder einzelnen Stimme eine diatonische Skala, die, bezogen auf die oberste Stimme, als E-lydisch (mit eigenem Leitton zur Terz) deutbar ist. Die Quartparallelen sind in Form von Vorschlägen präsent.

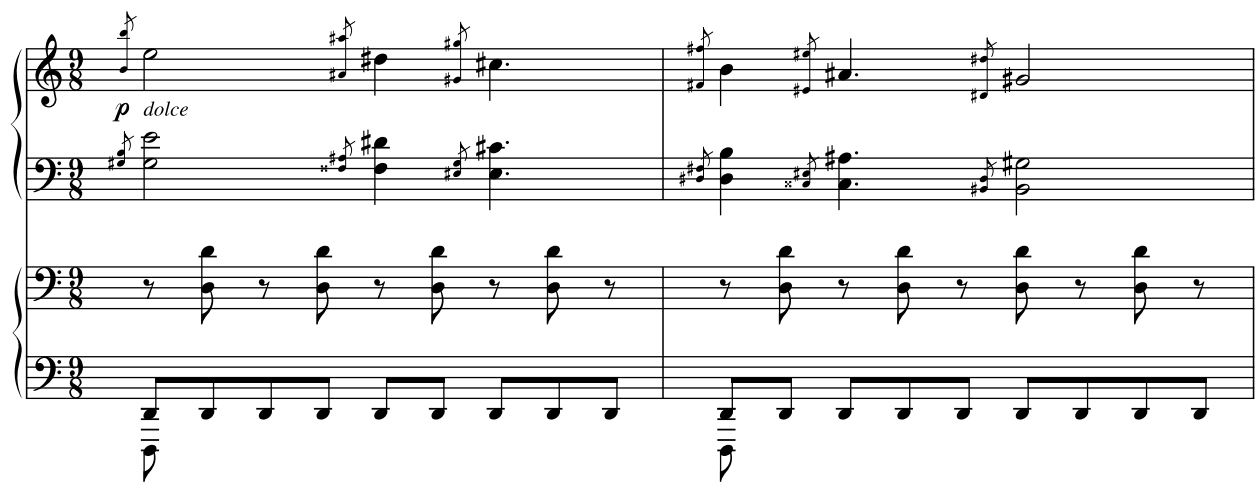

Beispiel 2: Béla Bartók, Sonate für zwei Klaviere und Schlagzeug, T. 84

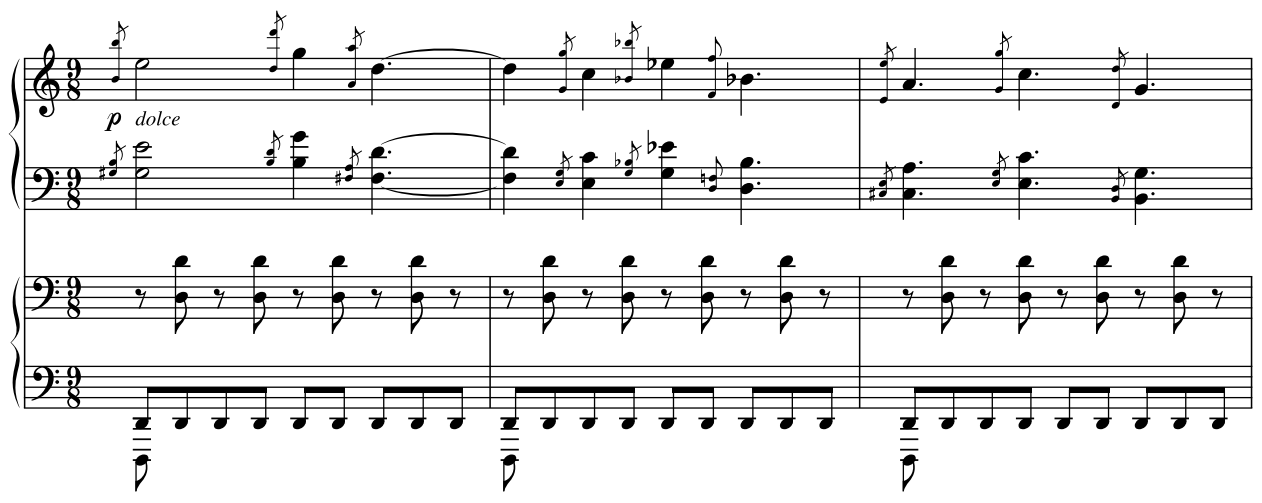

Beispiel 3: Béla Bartók, Sonate für zwei Klaviere und Schlagzeug, T. $88 \mathrm{ff}$.

4 Vgl. z. B. Werckmeister 1697, 7. 
Ein zweiter Abschnitt der Melodie verläuft in Sekunden, Terzen und Quarten. Erkennbar sind hier zwei bei Bartók übliche Verfahrensweisen: Zum einen die Verwendung diatonischer Skalen, auch mehrerer gleichzeitig. Hier wird die Bezugsskala E-lydisch mit Tönen aus verschiedenen anderen Skalen konfrontiert. Das repetierte $d$ der Begleitung entstammt E-mixolydisch, der zweite Abschnitt der Melodie bringt Töne aus E-äolisch und E-lokrisch. Mit Ausnahme des $f$ ist die gesamte chromatische Skala vertreten. Diese Chromatik ist jedoch nicht als Alterationschromatik oder angestrebte Dodekaphonie zu verstehen, sondern, mit einem von Bartók selbst verwendeten Begriff, als spolymodale Chromatikı.

Das andere dem Abschnitt zugrunde liegende Verfahren ist die Konstruktion von melodischem Material durch selbstgewählte Prämissen. Sekund- und (auch verminderte) Quartintervalle sind bereits die Basis des in der langsamen Einleitung auskomponierten Hauptmotivs.

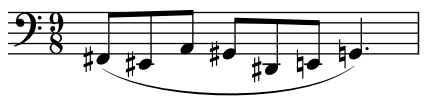

Beispiel 4: Béla Bartók, Sonate für zwei Klaviere und Schlagzeug, Einleitung

Doch noch auf andere Weise ist die Fauxbourdonpartie mit dem übrigen Tonsatz verzahnt. So tauchen bereits in der Einleitung parallelgeführte kleine Sexten auf.

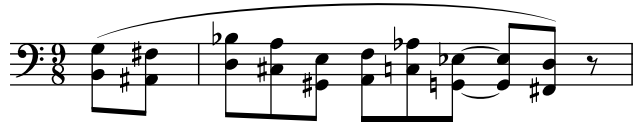

Beispiel 5: Béla Bartók, Sonate für zwei Klaviere und Schlagzeug, Einleitung, T. 9

Quartparallelen finden sich im ersten Thema als Begleitfigur.

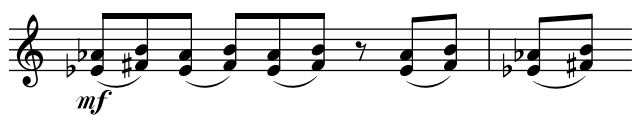

Beispiel 6: Béla Bartók, Sonate für zwei Klaviere und Schlagzeug, T. 41

Auch mit der nachfolgenden, thematisch eigenständigen Schlussgruppe ist die Fauxbourdonpartie durch das Sextintervall eng verbunden. Die Schlussgruppe basiert auf einem großen Sextsprung. Dieser wird über dem letzten H-Dur-Sextakkord des zweiten Themas eingeführt, so dass Dur- und Mollterz gleichzeitig erklingen - ein bei Bartók häufig anzutreffender Klang. ${ }^{5}$

5 Als theoretische Herleitung kann Polymodalität oder auch die oktatonische Halbton-Ganzton-Skala dienen. 


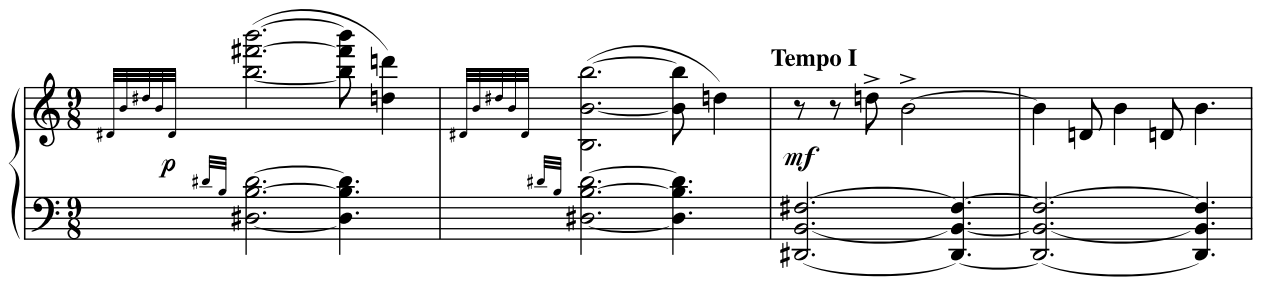

Beispiel 7: Béla Bartók, Sonate für zwei Klaviere und Schlagzeug, T. $99 \mathrm{ff}$.

Die zweite, kürzeste Fauxboudonstelle, T. 161 ff., erklingt im Unterquintkanon. Die zugrundeliegende Skala ist nun nicht mehr diatonisch, sondern besteht aus der chromatischen Ausfüllung eines Großterzrahmens. Es ergibt sich eine Spiegelstruktur, denn abwärts folgt eine kleine auf eine große Sekunde, aufwärts umgekehrt. Die ursprünglich bis zur Sexte aufstrebende diatonische Skala auf einen chromatischen Terzrahmen zu stauchen, entspricht dem Bartókschen Verfahren der >compression of range.$^{6}$

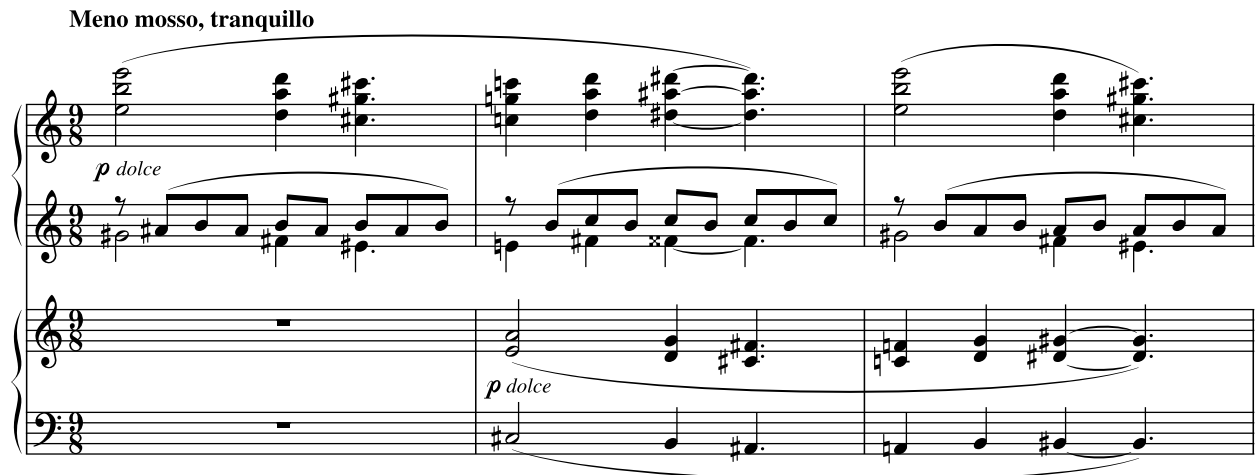

Beispiel 8: Béla Bartók, Sonate für zwei Klaviere und Schlagzeug, T. 161 ff.

Wieder ist die Fauxbourdonpartie in der vorausgehenden Passage vorbereitet. Es erklingen lange Ketten paralleler kleiner Sexten in Umkehrungsimitation. Auf den stranquillorAbschnitt folgt wiederum - in einer auf der oben erwähnten Begleitfigur (Beispiel 6) basierenden Durchführungspassage - eine Kette paralleler Quarten, die teils imitatorisch, teils komplett parallel in bis zu siebentönigen Quartenakkorden geführt werden. Die Fauxbourdonpartie ist also eingerahmt von Abschnitten, die auf den Fauxbourdonkomponenten sSextparallelen

In der Reprise, T. 292 ff., steht der dritte und längste Fauxbourdonabschnitt. Die Melodie ist als Umkehrung derjenigen der Exposition zu verstehen.

6 Entsprechend existiert auch die sextension of range «. Das Verfahren hat Bartók in seinen Harvard Lectures selbst beschrieben. 


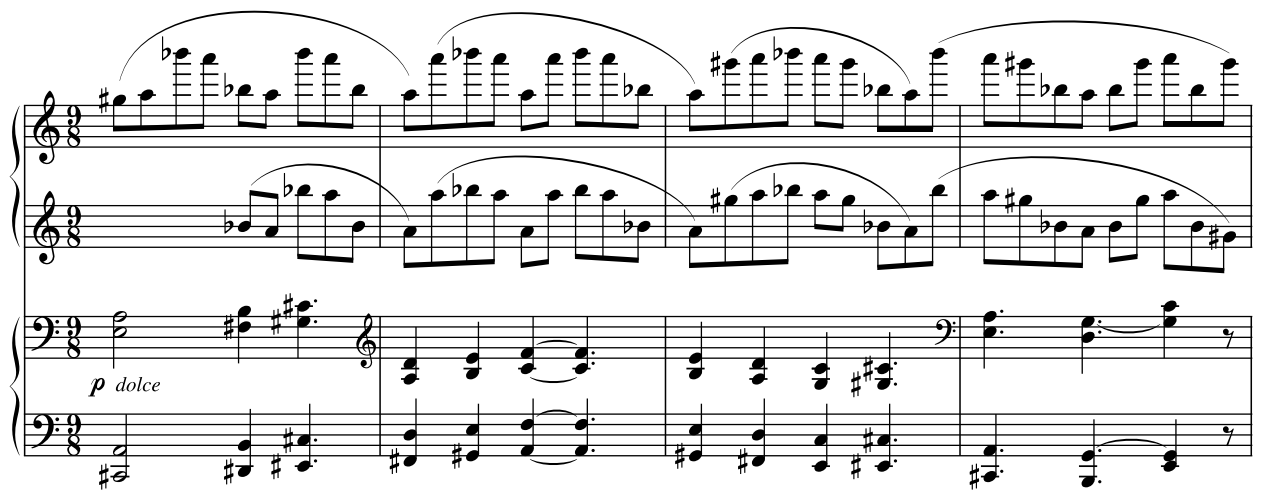

Beispiel 9: Béla Bartók, Sonate für zwei Klaviere und Schlagzeug, T. 292 ff.

Das zweite Klavier stellt die Melodie neun Takte lang vor, dann übernimmt sie acht Takte lang das erste Klavier eine große Sexte höher (bzw. kleine Terz tiefer). Im zweiten Klavier erklingt dazu das Thema im Umkehrungskanon, also in der (nicht intervallgetreuen) Gestalt des ersten Auftretens. Die Skala bezieht sich auf den Grundton a (ionisch/äolisch). Damit trägt Bartók dem Konzept des Sonatensatzes Rechnung, den Seitensatz in der Reprise eine Quinte tiefer zu bringen.

Vorbereitet ist die Fauxbourdonpassage durch die Quint-Oktav-Mixturen der beginnenden Reprise - es fehlt nur noch die Unterterzung zum Fauxbourdon, wie sie z. B. in T. $303 \mathrm{ff}$. erscheint. Am Ende des Abschnitts wird ein Teil der Melodie, ohne Fauxbourdon, im Ganztonabstand sequenziert, bis die vollständige Ganztonleiter durchlaufen ist (T. 309 ff.).

Auch hier, wie in der Exposition, mündet der Seitensatz in die Schlussgruppe. Vorher wird jedoch, ohne Mixturen, der Bogen zum zweiten Erscheinen des Fauxbourdoncharakters geschlagen: Die auf chromatische Schritte gestauchte Melodie erscheint, wiederum im Kanon.

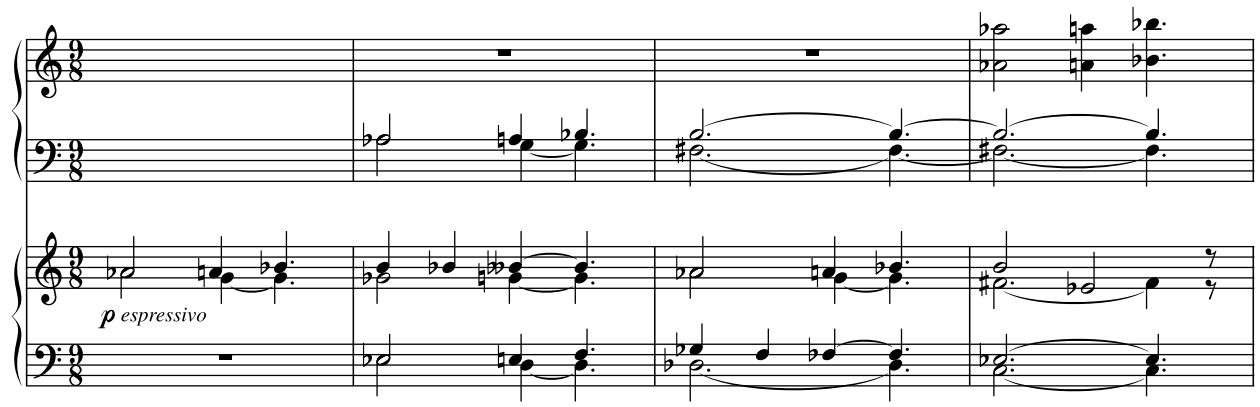

Beispiel 10: Béla Bartók, Sonate für zwei Klaviere und Schlagzeug, T. 321 ff. 
Es zeigt sich, dass anhand der Analyse eines aus der Kompositionsgeschichte überkommenen Details viel Grundsätzliches über Bartóks Schaffensweise verdeutlicht werden kann. Hierzu gehören die kontrapunktischen Verfahren des Kanons und der Intervallumkehrung, die Bartók von Bach, aber auch aus noch älteren musikalischen Sphären übernommen haben könnte. Deutlich wird darüber hinaus eine von Bach über Beethoven bis in die 2. Wiener Schule reichende Sensibilität für die Integration musikalischer Elemente, hier durch ımotivische Arbeit an kleinsten Details des Tonsatzes wie dem Quartintervall. Bartóks Arbeit am Material ist dabei sehr modern im Umkehren, Dehnen und Stauchen der möglichen Konstellationen. Die Verwendung überkommenen musikalischen Materials wie diatonischer und symmetrischer Skalen (z. B. der Ganztonleiter) in verfremdenden Kontexten ist ebenfalls typisch für Bartók.

Ein für die Sonate (insbesondere auch im Gegensatz zum Konzert für Orchester) charakteristischer Aspekt muss hier noch beleuchtet werden. Die Fauxbourdonpassagen in der Sonate für zwei Klaviere und Schlagzeug sind in hohem Grade intertextuell. Schon für den oberflächlichen Blick zu erkennen ist der Bezug zum Seitenthema aus Beethovens Waldstein-Sonate op. 53, der in der Übernahme nicht nur von Kontur und Charakter, sondern des gesamten Tonartenplans des Stücks besteht.

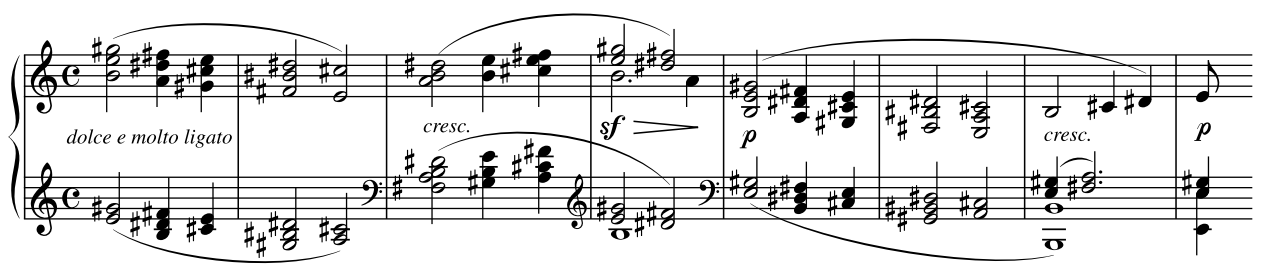

Beispiel 11: Ludwig van Beethoven, Klaviersonate C-Dur op. 53, I. Satz, T. 15 ff.

Bei Bartók finden so zwei Elemente aus der Musikgeschichte zusammen: Der ıweiche Charakter des Fauxbourdon und der sweiche`Charakter des Seitensatzes einer Sonate.

Auf ähnliche Weise verweisen die Fauxbourdonpassagen in der Sonate für zwei Klaviere und Schlagzeug auf J. S. Bach, wenn auch auf kein Einzelwerk im Speziellen. Man vergleiche Beispiel 8 mit der folgenden Passage aus Bachs Wohltemperiertem Klavier:

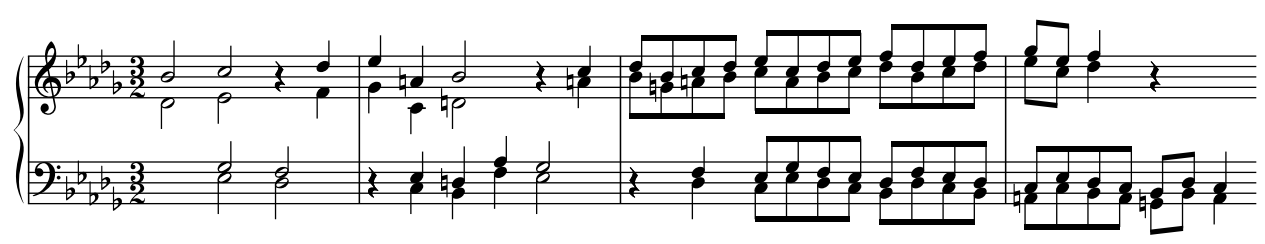

Beispiel 12: J. S. Bach, Fuge b-Moll, Das Wohltemperierte Klavier, Bd. II, T. $96 \mathrm{ff.}$

Hier wie dort haben wir einen Kanon bereits gekoppelter Stimmen. Nur dass in einer Bach-Fuge aufgrund der kontrapunktischen Selbständigkeit aller Stimmen nicht von 
`Kopplung` oder gar `Mixtur` gesprochen werden kann, sondern von einem `Canon sine pausis`, also einem Kanon ohne Einsatzabstand. Das Prinzip `Canon sine pausis` wiederum ist ursprünglich untrennbar mit dem Fauxbourdon verbunden, handelte es sich doch bei Dufays Kanontechnik sau Fauxbourdon` um einen Unterquartkanon ssine pausis`. Durch das Denkmodell `canon sine pausis` konnte der Fauxbourdonsatz in der Musikästhetik der Dufay-Zeit gerechtfertigt werden, die die Parallelführung von Stimmen über längere Strecken eigentlich nicht mit ihrem Varietas-Ideal vereinbaren konnte. In Bachs Fugentechnik ist nun ausgerechnet das Quartintervall, auch mit Unterterzung, vom `Canon sine pausis` ausgeschlossen. ${ }^{7}$ So finden bei Bartók Fauxbourdon und Kanon wieder zusammen, wobei der Fauxbourdon seinen kontrapunktischen Charakter als zweistimmiger Satz plus Kanon (sine pausis) zwar zugunsten der Mixtur verliert, er aber, eine Ebene höher, als Ganzes an kontrapunktischen Prozessen wieder teilhat.

\section{Konzert für Orchester}

Das Konzert für Orchester enthält einen Satz, der für das Thema `Mixtur bei Bartókı von besonderer Bedeutung ist: Der II. Satz mit dem Untertitel Giuoco delle Coppie (Spiel der Paare) führt in verschiedenen Intervallen gekoppelte Stimmpaare nacheinander vor. Dabei geht er fast alle Intervalle durch, in der Reihenfolge ihres Auftretens: (kleine) Sexte, (kleine) Terz, (kleine) Septime, Quinte, (große) Sekunde. Neben dem Einklang und der Oktave, die als Kopplung wahrscheinlich wegen ihrer Chroma-Identität ausgeschlossen werden, fehlt einzig die Quarte. Daher ergibt sich auch im zweiten Teil des Satzes, wenn zu den Stimmpaaren noch weitere parallele Stimmen hinzutreten, niemals eine traditionelle Sextakkordmixtur. Es scheint, als würde hier, in einer Art Paradestück zum Thema 'Mixturı, der Fauxbourdon absichtlich vermieden. An einigen Stellen jedoch nähert sich Bartók der Fauxbourdon-Faktur an, zum Beispiel in T. 212, wo die Quintenmixtur der Flöten durch die Terz ergänzt wird. So ergibt sich eine Sextakkordmixtur, wenn auch mit Quint- statt Quartparallelen (Beispiel 13).

Im Gegensatz zum Fauxbourdon kommen Mixturen von Durakkorden in Grundstellung häufig vor. Interessant sind die Durakkord-Mixturen als flirrende Klangflecken im Tremolo der Geigen in T. 241 ff. (Beispiel 14). Hier wird der vertraute Durakkord, ein Grundbaustein tonaler Musik, in seiner Verwendung als Mixtur und Tremolo Teil eines avantgardistischen Klangeffekts.

Auch in den anderen Sätzen des Konzerts für Orchester sind Durakkordmixturen anzutreffen. Weitaus am beliebtesten ist die Mixtur des Quartsextakkords. ${ }^{8}$ Auf Faux-

7 Ohne eine vierte Gegenstimme widerspräche ein solches Verfahren der polyphonen Fugenästhetik.

Eine solche vierte Stimme zu schreiben, ist jedoch innerhalb der Dissonanzbehandlungs- und Parallelführungsregeln kaum möglich.

8 Die Quartsextakkord-Mixtur scheint die Komponisten am Epochenumbruch zur Neuen Musik klanglich gereizt zu haben. Er spielt nicht nur bei Bartók, sondern auch (um nur wenige Beispiele zu nennen) bei Debussy und Messiaen eine wichtige Rolle. Der Quartsextakkord ist als Klang vertraut, je nach zugrundegelegtem Regelwerk auch konsonant, aber als Mixtur von allen DurakkordUmkehrungen am wenigsten verbraucht - die Sextakkordmixtur hat die Fauxbourdon-Tradition, die Mixtur der Grundstellung weckt ethnomusikalische bzw. mittelalterliche Assoziationen. 


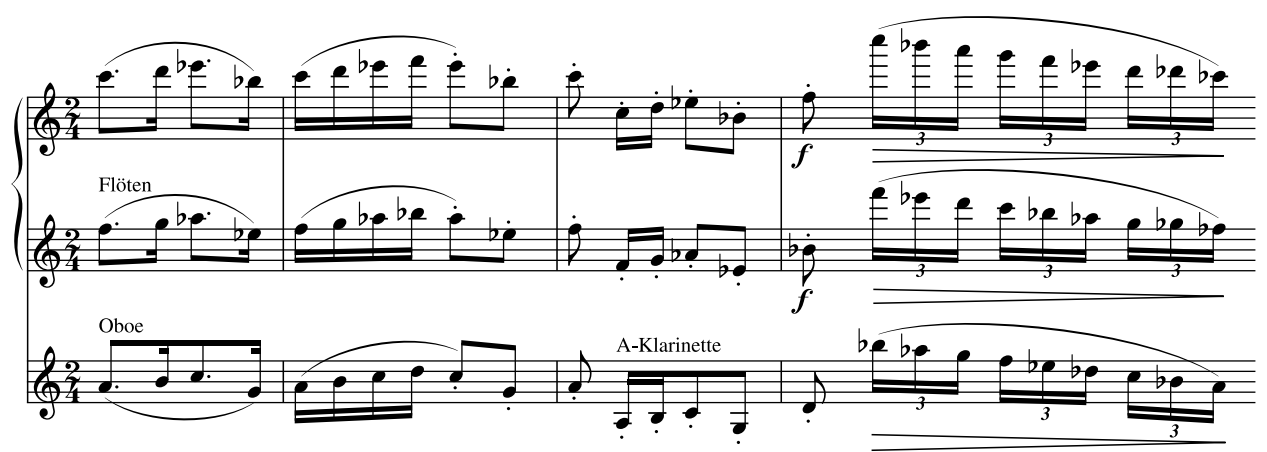

Beispiel 13: Béla Bartók, Konzert für Orchester, Giuoco delle Coppie, T. $212 f f$.

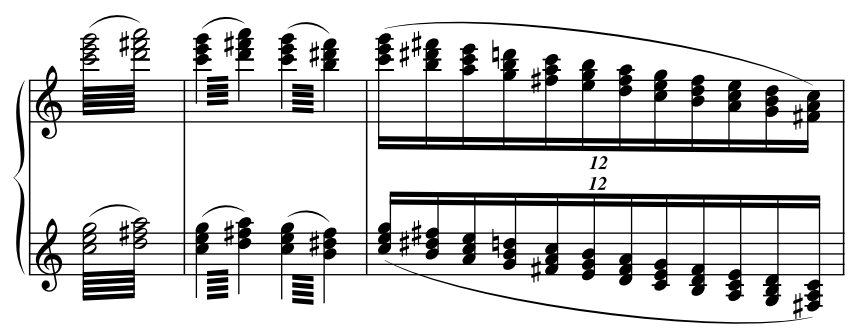

Beispiel 14: Béla Bartók, Konzert für Orchester, Giuoco delle Coppie, T. $241 f f$.

bourdonpassagen müssen wir bis zum Finale warten. In der Art eines Fugatos (ohne exakte motivische Übereinstimmung) setzen die Violinen nacheinander mit rasanten Sechzehntelketten ein. Die Stimmen verlaufen mit dem zweiten Einsatz in Terzparallelen und endlich, mit dem dritten Einsatz, in parallelen Sextakkorden. Eine vierte, tiefste Geigenstimme spielt in der Umkehrung mit.
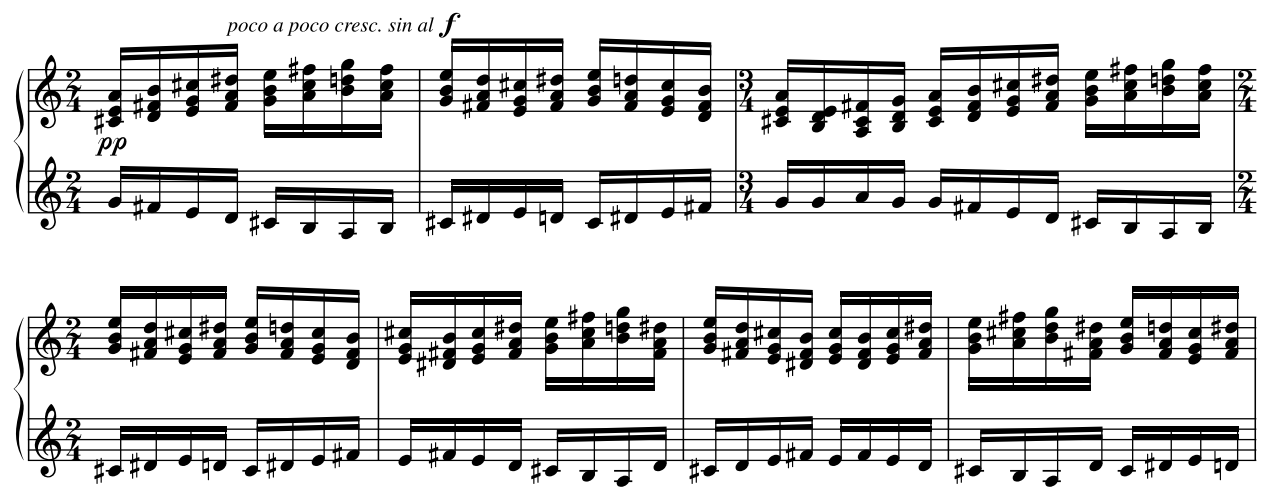

Beispiel 15: Béla Bartók, Konzert für Orchester, Finale, T. 21 ff. 
An dieser Konstellation hält Bartók immerhin dreizehn Takte, mehr als hundert Akkorde lang, fest, um im Folgenden mit den Kopplungen und Spiegelungen, unter Beibehaltung desselben Gestus, freier umzugehen. Überhaupt fällt als Charakteristikum von Bartóks Umgang mit Mixturen wie auch mit anderen zugrundegelegten Prinzipien auf, dass er sich jederzeit vorbehält, von innen abzukommen. Dies erschwert zwar die Analyse, bereichert aber das musikalische Erlebnis. Typisch ist beispielsweise hier im Finale die Passage T. 44 ff., der zwar ein Mixturgedanke klar zugrunde liegt; Umkehrung und Akkordtypen wechseln jedoch.

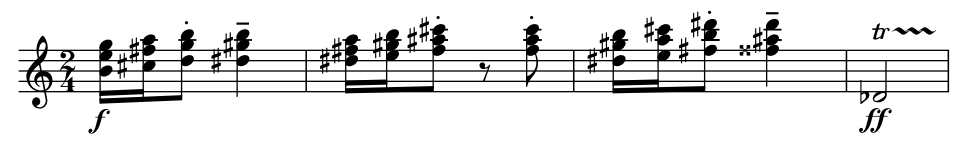

Beispiel 16: Béla Bartók, Konzert für Orchester, Finale, T. 44 ff.

Auf eine weitere Stelle sei noch das Augenmerk gelenkt, selbst wenn hier wieder keine Sextakkorde, sondern Quartsextakkorde parallel verschoben werden. Die Takte $175 \mathrm{ff}$. verweisen dennoch, wie die entsprechenden Abschnitte in der Sonate für zwei Klaviere und Schlagzeug, auf den Fauxbourdon, denn sie prägen, im Gegensatz zu der Sextakkordstelle T. $21 \mathrm{ff}$., den historischen sweichen s piano-dolce-Charakter des Fauxbourdon aus. Weitere Gemeinsamkeiten mit der Sonate für zwei Klaviere und Schlagzeug wie auch mit anderen Fauxbourdonstellen bei Bartók (z. B. der in Beispiel 1 zitierten Nr. 153 aus dem Mikrokosmos) sind die reale Mixtur und die dissonierenden Haltetöne.

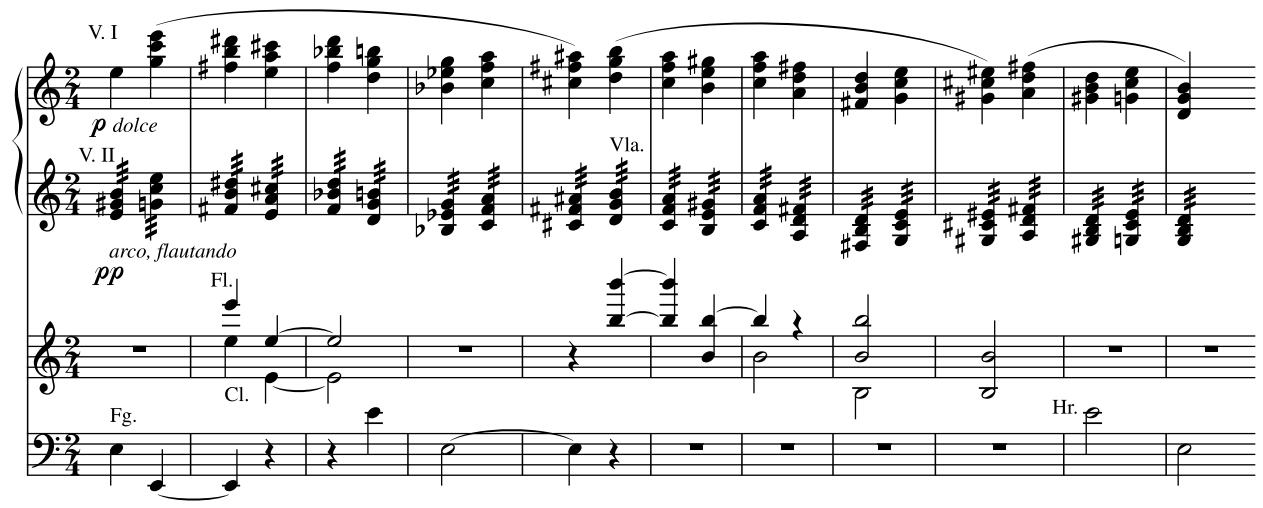

Beispiel 17: Béla Bartók, Konzert für Orchester, T. 175 ff.

Die beiden herangezogenen Stücke zeigen: Ihre Vergangenheit als Fauxbourdon macht die Verwendung der Sextakkordmixtur im 20. Jahrhundert problematisch. Sie wird daher häufig ausgespart, wie im Giuoco delle Coppie. Die Quartsextakkord-Mixtur kann als konsonanter und dennoch unverbrauchter Klang an ihre Stelle treten. 
Soll die Sextakkordmixtur dennoch zum Einsatz kommen, so gibt es für Bartók grundsätzlich zwei verschiedene Wege. Der erste besteht darin, sich auf die FauxbourdonTradition zu beziehen; In diesem Fall, wie in der Sonate für zwei Klaviere und Orchester, ist die Sextakkordmixtur als do/ce-Charakter inszeniert. Der hohe Konsonanzgrad der Stelle fällt auf, und geübte Hörer werden sogar die Verbindung zu älteren Fauxbourdonfakturen herstellen. Es soll hier nicht verschwiegen werden, dass die ıWeichheitı unter den historischen Charakterisierungen des Fauxbourdon ein Nebenaspekt war. Deutlich häufiger und durchgehender ist in den Theoretikerquellen von »Traurigkeit« und »Mattigkeit» die Rede, was mit einer nachträglichen Ausdeutung der Wortkomponente sfaux zusammenhängen mag. Es ist also bei Bartók bei aller strukturellen Kontinuität eine konnotative Verlagerung in der Semantik (von straurig/matt zu ıweich`) zu konstatieren. Die semantische Aussage von Instrumentalmusik ist im Detail schwer zu belegen, aber es scheint, als habe diese Verlagerung spätestens im 18. Jahrhundert stattgefunden und bei den zeitgenössischen Musikschriftstellern keinen ausreichenden Niederschlag gefunden.

Die zweite Möglichkeit der Verwendung von Sextakkordmixturen bei Bartók, die ich in diesem Zusammenhang nicht `Fauxbourdon` nennen würde, ist eine nicht-intertextuelle. So wie im letzten Satz des Konzerts für Orchester können sich Sextakkordmixturen quasi akzidentiell aus den konstruktiven Kompositionsprinzipien ergeben. In diesem Fall, ohne die Inszenierung ihrer historischen Konnotationen, ist die Sextakkordmixtur trotz ihrer beträchtlichen Dauer und Konsequenz auditiv nicht oder kaum als `Fauxbourdon` wahrnehmbar.

\section{Literatur}

Bartók, Bela (1931), „Vom Einfluss der Bauernmusik auf die Musik unserer Zeit«, in: Béla Bartók, Weg und Werk. Schriften und Briefe, hg. von Bence Szabolcsi, Kassel u.a.: Bärenreiter 1972, 175.

Bartók, Bela (1981), „Revolution und Evolution in der Kunst», in: Bela Bartók (= MusikKonzepte 22), hg. von Heinz-Klaus Metzger u. Rainer Riehn, München: Edition Text + Kritik.

Besseler, Heinrich (1950), Bourdon und Fauxbourdon, Leipzig: Breitkopf \& Härtel.

Fladt, Hartmut (1996), „Bela Bartók«, in: Lexikon der Komponisten der Gegenwart, hg. von Hans-Werner Heister u. Wolfgang Sparrer, München: Edition Text + Kritik.

(2005), »Modell und Topos im musiktheoretischen Diskurs. Systematiken/Anregungen«, Musiktheorie 20, 343-369.

Hoffmann-Axthelm, Dagmar (1973), Art. »Fauxbourdon« in: Handwörterbuch der musikalischen Terminologie, hg. von Hans Heinrich Eggebrecht, Wiesbaden: Steiner.

Jeßulat, Ariane (2001), Die Frage als musikalischer Topos (= Berliner Musik-Studien 21), Sinzig: Studio. 
Szabolcsi, Bence (1972), Béla Bartók. Weg und Werk. Schriften und Briefe [1957], Kassel u. a.: dtv/Bärenreiter.

Werckmeister, Andreas (1697), Hypomnemata musica, Quedlinburg, Reprint Hildesheim: Olms 1994. 